\title{
Advanced Molecular Biologic Techniques in Toxicologic Disease
}

\author{
Jeanine Ward • Gyongyi Szabo • David McManus • \\ Edward Boyer
}

Published online: 10 November 2011

(C) American College of Medical Toxicology 2011

\begin{abstract}
The advancement of molecular biologic techniques and their capabilities to answer questions pertaining to mechanisms of pathophysiologic events have greatly expanded over the past few years. In particular, these opportunities have provided researchers and clinicians alike the framework from with which to answer clinical questions not amenable for elucidation using previous, more antiquated methods. Utilizing extremely small molecules, namely microRNA, DNA, protein, and nanoparticles, we discuss the background and utility of these approaches to the progressive, practicing physician. Finally, we consider the application of these tools employed as future bedside point of care tests, aiding in the ultimate goal of unsurpassed patient care.
\end{abstract}

Keywords Molecular techniques · MicroRNA .

Hepatotoxicity · Early markers

J. Ward $(\bowtie) \cdot$ E. Boyer

Department of Emergency Medicine, University of Massachusetts

Medical School,

55 Lake Avenue North,

Worcester, MA 01655, USA

e-mail: jeanine.ward@umassmemorial.org

G. Szabo

Division of Hepatology; Department of Medicine,

University of Massachusetts Medical School,

55 Lake Avenue North,

Worcester, MA 01655, USA

D. McManus

Departments of Medicine and Quantitative Health Science,

University of Massachusetts Medical School,

55 Lake Avenue North,

Worcester, MA 01655, USA

\section{Introduction}

Molecular mechanisms of toxicity have not been rigorously established for many xenobiotics. In this manuscript, we will describe advanced molecular biological approaches to the assessment of genetic, translational, molecular, and regulatory mechanisms that operate in overdose or acute toxicologic processes. We will discuss recent technologies that can be directed toward bedside point of care testing as well as describe how these innovative methods may help identify early serum biomarkers of toxicity for a variety of xenobiotics. Medical toxicologists should consider applying these advanced molecular approaches to advance our current understanding of hepatotoxicity and to improve the identification and treatment of at-risk patients.

\section{Terminology}

Genomics

Genomics is the study of genetics pertaining to the similarities and differences among the genomes of organisms (Table 1) [1]. Genomics links genetic variability (e.g., genotype) with the physical appearance of an individual (e.g., phenotype). The genetic makeup of an individual therefore drives the susceptibility to disease, from schizophrenia to coronary artery disease [2]. Genomics techniques often involve identifying single nucleotide polymorphisms (SNPs), commonly occurring DNA sequence variations in members of the same species. Highthroughput DNA-sequencing techniques have dramatically expanded analysis of databases to find associations between 
Table 1 Advanced molecular biologic techniques: definition of terms

\begin{tabular}{|c|c|}
\hline Terms & Definitions \\
\hline Genomics & Study of the genetic material or "blueprint" found in living organisms \\
\hline $\begin{array}{l}\text { Genome-Wide Association } \\
\text { Study (GWAS) }\end{array}$ & Genetic approach (typically using microarray technology) to determine unique individual DNA sequences \\
\hline Metabolomics & $\begin{array}{l}\text { The study of variation of metabolites (typically using GC-MS technology) to determine metabolite variation } \\
\text { usually in relation to disease processes }\end{array}$ \\
\hline Microarray & $\begin{array}{l}\text { Study of thousands of genes or proteins simultaneously. Typically, the sample of interest (i.e., DNA) is placed } \\
\text { on a glass slide or "chip." Fluorescently labeled known samples are then allowed to bind to the chip, where } \\
\text { the degree of resultant fluorescence is indicative of the presence and amount of known sample }\end{array}$ \\
\hline MicroRNA & Short, non-coding regions of mRNA which regulate gene translation, usually via inhibition \\
\hline Pharmacogenomics & The study of variation of DNA and/or RNA transcripts in relation to drug exposure \\
\hline Toxicogenomics & $\begin{array}{l}\text { The study of variation of DNA and/or RNA (usually using microarray technology) to determine transcript } \\
\text { variation in relation to disease processes }\end{array}$ \\
\hline Transcriptomics & $\begin{array}{l}\text { The study of variation of DNA and/or RNA (usually using microarray technology) to determine transcript } \\
\text { variation in relation to normal development or disease processes }\end{array}$ \\
\hline GC-MS & Gas chromatography-mass spectrometry \\
\hline
\end{tabular}

patterns of SNPs and specific disease states prevalent in patient subpopulations. Because genetic mutations can be identified in regions as small as $10 \mathrm{~kb}$, SNPs provide an efficient means to identify unique genes associated with diseases or adverse drug events [2].

Genomic techniques have immediate applicability to the field of medical toxicology. For example, medications such as methadone and antipsychotics are associated with QTc prolongation, torsades de pointes, and death. Genome-wide association studies (GWAS) have identified unique genes which place certain individuals at risk for QTc prolongation development. One specific SNP associated with QTc prolongation is nitric oxide synthase 1-AP (NOS1AP). NOS1AP binds to the intracellular domain of the membrane-associated guanylate cyclase protein synapse-associated protein 97 (SAP97). SAP97, in turn, co-localizes intracellularly with the inwardly rectifying potassium channel, which is responsible for repolarization of the action potential. These data suggest that NOS1 AP is potentially involved with cardiac repolarization and resultant QTc prolongation [3].

Genomic methods have also been applied to the evaluation of molecular mechanisms of addiction. A GWAS study has proven effective in investigating an association between specific alcohol dehydrogenase (ALDH) haplotypes within four distinct alcohol-dependent populations [4]. This investigation of American Indians, Southwest American Indians, Finnish Caucasions, and African Americans identified an association of ALDH isozymes, namely 1A1 and 4, in Southwest American Indian populations [5]. Genomics may therefore help identify molecular targets whose modification may alter the development of substance abuse progression.

\section{Transcriptomics}

The study of gene transcripts or transcriptomics (Fig. 1) frequently utilizes microarray assays of complementary deoxyribonucleic acid expression to identify up- or downregulation of specific protein encoding messenger ribonucleic acid (mRNA) oligonucleotides [6]. Microarray technology involves adhering sequence-specific oligonucleotides or proteins in a prearranged fashion to a solid surface, such as glass. These solid surfaces, often times referred to as microarray "chips" are small, being no more than a few centimeters in diameter, and are used in highthroughput testing. The oligonucleotides or proteins to be evaluated are first enzymatically cleaved. They are then labeled with fluorescent markers and incubated with the microarray chip. After chip washing, the intensity of the resultant fluorescent signaling is indicative of transcript or protein binding [7].

Microarray technology allows thousands of genes to be analyzed simultaneously. This method represents a major technical advancement and is the framework within which drug therapy discovery often occurs $[4,8]$. Up to $90 \%$ of the base pairs in mammalian DNA are non-protein coding. Identifying a downstream product of the gene transcription process can therefore produce a more focused assessment of potential targets. A transcriptomic approach can accelerate the discovery of many target gene transcripts associated with toxicologic conditions and determine the mechanism of drug action and metabolism. For instance, microarray technology has been used to investigate the mechanism of action for A-972611, a novel human immunodeficiency virus (HIV) protease inhibitor. This study revealed an association with A-972611 and CYP3A4 transcript reduction. A-972611 


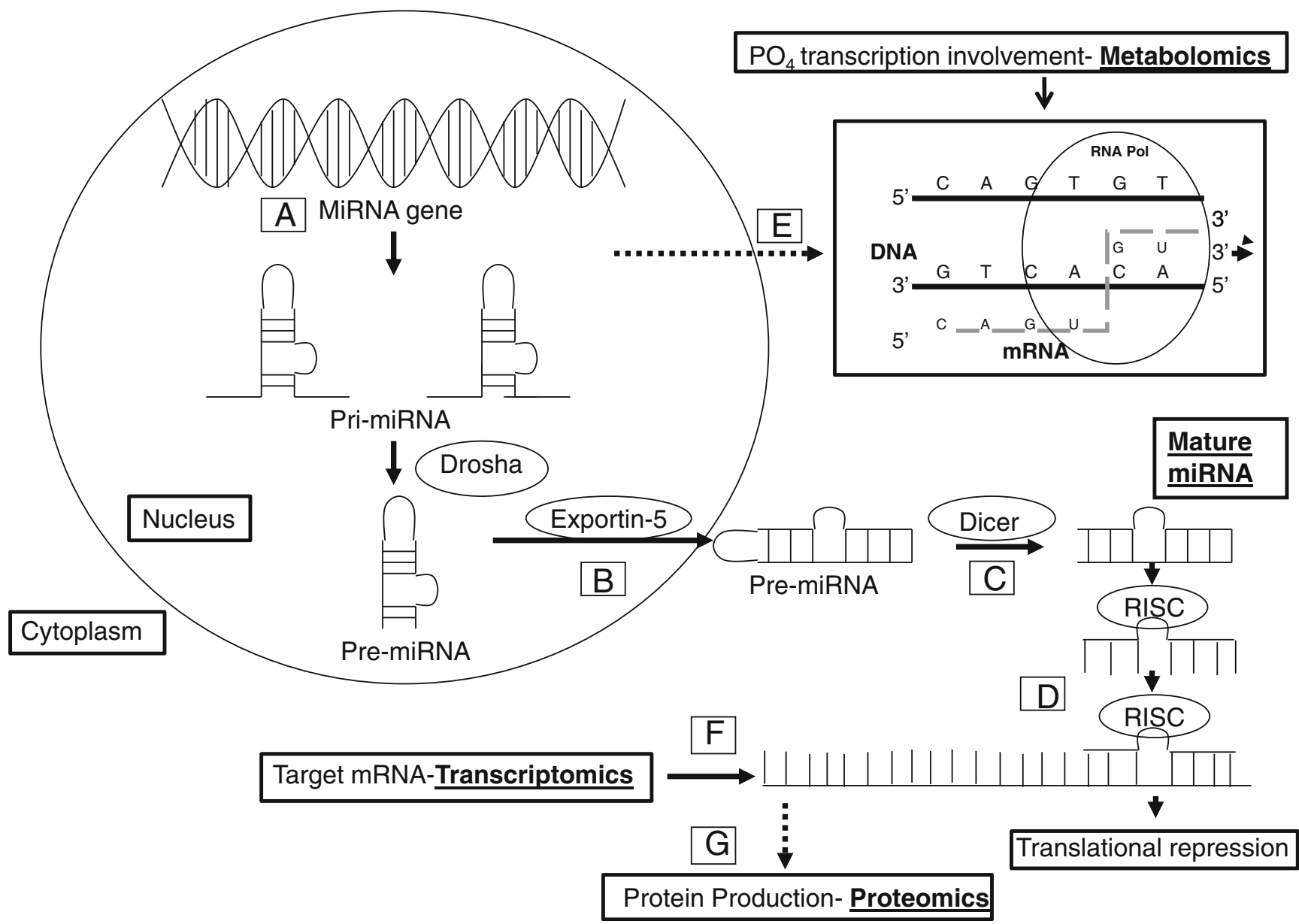

Fig. 1 Mechanisms of nucleic acid processing. $(A)$ Intranuclear miRNA initial transcription to primary (Pri)-miRNA form, cleaved by Drosha to precursor (Pre)-miRNA. (B) Pre-miRNA translocation to the cytoplasm by the exportin-5 protein. $(C)$ Pre-miRNA cleavage by the endoribonuclease protein, Dicer to mature miRNA. $(D)$ Mature miRNA transcript loading of RNA-induced silencing complex (RISC)

also downregulates the normal rifampin activation of CYP3A4. Because rifampin activates the nuclear receptor and transcription factor known as pregnane-X receptor (PXR) in human hepatocytes, it was hypothesized that A-972611 inhibits rifampin PXR activation via the CYP3A4 pathways. This hypothesis was confirmed with A-972611 inhibition of PXR, with transcriptomics revealing a novel mechanism of action for this HIV protease inhibitor [9].

More specifically, the term pharmacogenomics describes the study of variations of DNA and RNA responses to drug exposure using GWAS studies or microarray technology [6,7]. Unique DNA and RNA responses to xenobiotic exposure may herald adverse drug reactions. For example, $1-14 \%$ of patients who receive abacavir for HIV may develop hypersensitivity reactions such as Stevens-Johnson's syndrome with HLA-B*5701 allele carriers being at increased risk [10]. Consequently, a polymerase chain reaction of the DNA of abacavir protein to target mRNA translational repression. $(E)$ The process of intranuclear transcription of DNA to mRNA. The identification of required phosphate $(\mathrm{PO} 4)$ metabolites in transcription is an example of metabolomics. Production of unique mRNA transcripts $(F)$ and proteins $(G)$ are examples of transcriptomics and proteomics, respectively

recipients followed by hydridization with sequencespecific oligonucleotide probes may identify susceptible individuals carrying the HLA-B*5701 allele (http://www. questdiagnostics.com/). Identification of individuals carrying the HLA-B*5701 allele precluded prescription of abacavir to suspectible patients. Importantly, this testing resulted in a decline of abacavir-associated adverse drug reactions from $7.8 \%$ to $3.2 \%$ [11].

The term toxicogenomics is similar to pharmacogenetics, but is unique in that it focuses on the genetic changes that occur specifically as a result of drug-induced cell or organ dysfunction and injury [12]. For example, bromobenzene hepatotoxicity was evaluated in a rat model using both the transcriptome and proteome approach. With bromobenzene exposure, numerous genes associated with glutathione (GSH) metabolism were shown upregulated, including gamma-glutamylcysteine synthetase, the rate-limiting enzyme involved in GSH synthesis. This approach is significant since 
it has been established bromobenzene metabolism results in 3,4 epoxide metabolite. The irreversible binding of 3,4 epoxide metabolite to GSH then causes resultant hepatotoxic effects [13].

\section{MicroRNAs}

MicroRNAs (miRNAs) are noncoding posttranslational regulators that bind to untranslated mRNA sequences producing gene silencing. Moreover, each miRNA targets several different mRNAs. Briefly, the miRNA gene is first transcribed into a primary microRNA transcript, then cleaved by the intranuclear Drosha protein into precursor microRNA (Fig. 1). Exportin-5 protein, a Randependent importin-beta-related transport receptor protein, then translocates pre-mRNA transcripts into the cytoplasm, where it is further cleaved by the endoribonuclease Dicer into mature microRNA segments. These segments are approximately 19-24 nucleotides in length. Dicer, a member of the ribonuclease III family, is also responsible for initiating the mature miRNA transcript binding to RNA-induced silencing complex (RISC), which together inhibits mRNA translational of target genes (Fig. 1). The same target gene may then be regulated by several different miRNAs in different biological situations, a process that allows enormous complexity and flexibility in regulatory potential. MicroRNA fragments, being short, chemically stable circulating biomolecules, may be ideal serum biomarkers, since they do not suffer from the common limitations of protein research such chemical instability and posttranslational modifications [8].

MiRNAs have been characterized as regulators of protein expression in diverse disease processes, including acute hepatic injury. A study of murine acetaminophen (APAP) intoxication identified highly significant differences in the spectrum and levels of miRNAs, including miRNA-122 in both liver and plasma between control and overdosed animals [8]. The liver-specific miRNA-122 has also been shown associated with hepatitis $\mathrm{C}$ and hepatocellular carcinoma disease progression. In addition, a correlation between has been implicated between miRNA-122 and other biological processes, such as controlling the expression of hepcidin, important in iron homeostasis. The exact role of miRNAs in hepatotoxicity, however, still remains to be determined [14].

Importantly, the ubiquity and structural stability of miRNAs allows the potential to correlate unique, timedependent miRNA "fingerprints" with specific intoxications. For example, miRNAs have been utilized as early biomarkers for numerous disease processes, including esophageal squamous cell carcinoma [15], Parkinson's disease [16], steatohepatitis [17], and hepatocellular carcinoma $[18,19]$. For instance, miR-375 and mir 92a were shown correlated with hepatocellular carcinoma (HCC) in hepatitis B virus (HBV) infected individuals. These miRNAs could uniquely distinguish between individuals infected with either HBV alone, hepatitis $\mathrm{C}$ virus, or nonviral-infected patients. This suggests miR-375 and mir92a can be used as effective biomarkers in establishing HBVassociated HCC from other virally induced hepatopathology [19]. Together, this demonstrates miRNAs may be successfully employed as an ideal area of research to determine other early biomarkers for disease states, notably APAP-associated hepatotoxicity [8]. This is significant since if validated, would confirm miRNA as a future novel plasma marker of APAP-induced hepatic damage.

\section{Proteomics}

Proteomics involves the study of downstream transcription products or the actual proteins coded by a particular DNA sequence (Fig. 1). By evaluating larger biomolecules, proteomics investigates both the structure and function of proteins using microarray techniques, as well as identifying unique peptides of interest compared to nucleotide transcripts. Because of protein folding, chemical modifications, and other posttranslational events, proteomic methods also utilize chemical analytical techniques, such as microarray 2-dimensional (2-D) gel electrophoresis and mass spectrometry [20].

Proteomic methods have immediate relevance to the study of toxicologic processes. For example, an experimental model of APAP toxicity involved the development of chimeric mice and human hepatocytes. First, investigators established control proteomic responses to APAP hepatotoxicity, then administering toxic doses of APAP to the chimeric mice. To confirm hepatotoxic effects in murine compared to human hepatocytes in these chimeric mice, investigators compared 2-D electrophoresis and mass spectrometry results for aldehyde dehydrogenase, ATP synthase alpha chain, and glutathione S-transferase in both cell types. The proteomic profile for human APAP hepatoxicity suggests that lipid/fatty acid metabolism and oxidative stress are involved in acetaminophen poisoning [20].

Proteomic methods can also provide new areas of investigation including toxic effects of nanomaterials. Carbon nanotubes (CNT), used in products as diverse as new bone growth scaffolding to ice hockey stick [21, 22], are structurally similar to asbestosis fibers [23]. To investigate carbon microtubules carcinogenic potential, researchers analyzed pulmonary tissue from mice exposed to CNT by pharyngeal aspiration for 3 weeks. Their results revealed upregulation of s100a9, a biomarker of asbestos exposure [24]. This study was the first to report the upregulation of s100a9 in a rodent model of inflammatory 
lung disease, suggesting the usefulness of this model in discovering novel biomarkers of inhalational toxicologic injury, including CNT. These results also support the utility and effectiveness of a proteomic approach in evaluating the physiologic responses to toxiocologic exposures [24].

\section{Metabolomics}

Metabolomics involves the simultaneous evaluation of thousands of metabolites utilizing liquid chromatographymass spectrometry or gas chromatography-mass spectrometry (GC-MS). With this approach, specific metabolites (usually the resultant small molecules of metabolism), their relation to proteins, their function as cofactors in metabolic processes, and their involvement in gene transcription and translation may be more clearly determined (Fig. 1). In addition, this approach can identify novel biomarkers from a variety of biological matrices such as serum, cerebral spinal fluid, saliva, and urine [25].

Metabolomics has been applied to the study of hepatoxic agent tetraethyl [(3-hydroxy-2-pyridyl)amino]methandiphosphonate (14SC), a known rat hepatotoxin, was administered to rats to specifically identify urine and serum biomarkers of hepatotoxicity. GC-MS metabolomics identified increased unconjugated bile acid accumulation in both urine and serum in this experimental model. The same study also found decreased coenzyme A ligation gene expression, an enzyme necessary for bile acid conjugation. These researchers therefore theorized that 14SC interferes with bile acid metabolism to process hepatic injury [26].

\section{Computational Systems Biology}

Computational systems biology is an advanced mathematical modeling approach using large amounts of biologic data. These systems use a high confidence network system integrating biology and chemical toxicology to estimate likely future adverse interactions. This approach can therefore prevent human morbidity and mortality by estimating the likelihood of adverse drug effects. For example, one statistical model based on known proteinchemical interactions is the Comparative Toxicogenomic Database (CTD). This system compares exposure to 2,490 chemicals and production of 6,060 proteins to identify 42,194 potential associations with specific adverse events [27]. For instances, di-ethylhexylphthalate (DEHP), a plasticizer for polyvinyl chlorate, is used in a variety of substances including intravenous tubing and nasogastric tubes. Metabolites of DEHP include phthalates, known endocrine disruptors that are associated with increased DNA damage in human sperm [28]. DEHP also inhibits connexin (gap junction) conduction in cardiomyocytes to produce arrhythmias in vitro [29]. Finally, DEHP has been implicated in neurologic defects in exposed developing fetuses [30]. Using the CTD approach to determine the role DEHP may play in these pathophysiologic events, numerous proteins had projected interactions with DEHP, including gamma-amino butyric acid (GABA) A receptors. This interaction has been supported in the literature with DEHP modulating GABA receptors function, similar to that of volatile anesthetics in experimental models [31]. Another nanotoxic environmental xenobiotic, 2,3,7,8 tetrachlorodibenzo-p-dioxin (TCDD), produced by incineration of industrial compounds, was projected via the CTD system to be associated with protein kinase $\mathrm{C}$ epsilon (PRKCE). PRKCE was then shown to be associated with brain tumor formation in a study evaluating TCDD neurotoxicity in cerebellar granule cells [32]. Therefore, the computational system biology approach may be useful in predicting specific toxicologic outcomes of specific exposures [27].

\section{Application of Advanced Technologies}

These novel molecular biologic approaches can be applied to the evaluation of hepatotoxicity. Among hepatotoxic drugs, APAP remains an important etiology of acute liver failure in the USA [33, 34]. Assessment of patients suspected of APAP ingestion can be difficult for treating physicians, since up to $24 \mathrm{~h}$ may elapse before signs of hepatic injury may appear. The need to admit patients while potential hepatotoxicity is assessed leads to elevated rates of hospital admissions [35]. Despite the prevalence of APAP poisoning and over 30 years of research, the exact mechanism of acetaminophen toxicity remains unclear [36].

The role of specific chemical intermediates in mediating hepatotoxicity is uncertain. For example, Nacetyl-p-benzoquinone imine (or NAPQI) is a reactive metabolite that is inactivated by glutathione at therapeutic APAP doses. NAPQI may covalently bind to cellular proteins following glutathione depletion to produce hepatocellular injury. The production of NAPQI alone, however, cannot entirely explain APAP-induced liver damage. Transgenic mice overexpressing glutathione synthetase which produce more glutathione paradoxically suffer greater hepatotoxicity than the wild-type mice at identical APAP dosing [37]. In addition, APAP can still cause hepatotoxicity without decreased glutathione levels in a mouse embryo development model [38], suggesting mechanism other than APAP is involved in causing liver damage.

Additional hepatotoxic pathways may involve the production of reactive oxygen species and inflammatory injury. Oxidants, including peroxides, promote mitochon- 
drial permeability transition (MPT) which increases entry of ions, uncoupling of oxidative phosphorylation, and decreased ATP synthesis. Cytokines IL-1 and IL-10 have been implicated in APAP-induced hepatotoxicity, as well as tumor necrosis factor alpha [39]. Interestingly, increased expression of the transcription factor hypoxia inducible factor 1-alpha (HIF-1-alpha) occurs after MPT upregulation in the setting of APAP toxicity and oxidative stress. This is significant since HIF-1-alpha targets upregulation of vascular endothelial growth factor, a biomolecule that stimulates angiogenesis and that has been associated with hepatocellular recovery and regrowth in the setting of hepatotoxicity [11].

An accurate early serum marker for APAP toxicity that is stable, specific, and easily measured still remains elusive. Standard clinical laboratory testing may not reveal evidence of hepatic injury for up to $24 \mathrm{~h}$ following APAP ingestion. A considerable proportion of APAP-exposed individuals therefore receive unnecessary empiric treatment with an antidote before hepatic injury can be eliminated. There is a need for earlier diagnostic indicators of APAP induced hepatic injury. We therefore intend to identify biochemically stable serum markers of APAP exposure that slows clinicians to accurately establish the diagnosis of APAP hepatotoxicity and its progression to acute liver failure. Unfortunately, most candidate biomarkers (e.g., protein adducts) possess physiochemical properties that limit their use in rapid diagnostic testing. MicroRNA fragments, used as early serum markers for other disease processes, do not suffer from these limitations [8]. In fact, using APAP hepatotoxicity as an example, a murine APAP intoxication study identified highly significant differences in the spectrum and levels of miRNAs in both liver and plasma between control and overdosed animals [8]. Unfortunately, this study lacked clinical and diagnostic relevance; it examined neither extended time dependency of miRNA concentrations nor extensive dose dependency of these important biomarkers of injury. Consequently, alterations in the "fingerprint" of miRNA concentrations that change with time in the setting of hepatotoxicity may be necessary to define injury.

\section{Future Directions}

The development of novel molecular biologic techniques may revolutionize the evaluation of the pathophysiology of xenobiotic toxicity. Specifically, transcriptomic, proteomic, and metabolomic technologic approaches would be beneficial in determining the specific pathophysiologic pathways of APAP toxicity, in turn providing numerous future therapeutic options. However, each of these processes may take days to weeks to complete. In addition, current pharmacogenetic tests may require days to weeks to complete and often require considerable laboratory technical skill to perform. Current pharmacogenetic methods are therefore inappropriate for use in acutely ill patients. Novel nanotechnology approaches, in use with resulted molecular biologic data, for example include microfluidic technology and dip pen lithography. These methodologies have the potential to yield real-time, bedside results with high sensitivity and specificity [40].

Of note, because the exact mechanism of injury for many hepatotoxins is unknown, novel technologies including determining unique "fingerprint" miRNA of hepatotoxicity can be further elucidated with these techniques. The impact of these techniques on improving patient care and lowering healthcare costs is of clear importance.

Conflict of interest The authors report no conflict of interest.

\section{References}

1. Bickel P, Brown JD, Huang H, Li Q (2009) An overview of recent developments in genomics and associated statistical methods. Phil Trans R Society 367(1906):4313-4337

2. Manolio TA, Collins FS (2009) The HapMap and genome-wide association studies in diagnosis and therapy. Annu Rev Med 60:443-456

3. Arking DE, Pfeufer A, Post W et al (2006) A common genetic variant in the NOS1 regulator NOS1AP modulates cardiac repolarization. Nat Genetics 38:644-651

4. Weinshilboum R, Wang L (2005) Pharmacogenomics: bench to bedside. Discovery Med 5(25):30-36

5. Liu J, Zhou Z, Hodgkinson C, Yuan Q, Shen P-H, Mulligan CJ, Wang A, Gray RR, Roy A, Virkkunen M, Goldman D, Enoch M-A (2011) Haplotype-based study of the association of alcoholmetabolizing genes with alcohol dependence in four independent populations. Alcohol Clin Exp Res 35(2):1-13

6. Wang L, McLeod H, Weinshilboum RM (2011) Genomics and drug response. NEJM 364(12):1144-1153

7. Feero WG, Guttmacher EG, Collins FS (2010) Genomic medicine - an updated primer. NEJM 362:2001-2011

8. Wang K, Zhang S, Marzolf B, Troisch P, Brightman A, Hu Z, Hood LE, Galas DJ (2009) Circulating microRNAs, potential biomarkers for drug-induced liver injury. PNAS 106:4402-4407

9. Blomme EAG, Yang Y, Waring JF (2009) Use of toxicogenomics to understand mechanisms of drug-induced hepatotoxicity during drug discovery and development. Toxicology Lett 186:22-31

10. Alfirevic A, Pirmahamed M (2010) Drug induced hypersensitivity reactions and pharmacogenomics: past, present, and future. Pharmacogenomics 11(4):497-499

11. Phillips EJ, Mallal SA (2009) Personalizing antiretroviral therapy: is it a reality? Per Med 6:393-408

12. Chaudhuri S, McCullough SS, Hennings L, Letzig L, Simpson PM, Hinson JA, James LP (2011) Acetaminophen hepatotoxicity and HIF-a-alpha induction in mice occurs without hypoxia. Tox and Applied Pharm 252(3):221-220 
13. Stierum R, Heijne A, Kienhuis B, Ommen V, Groten J (2005) Toxicogenomics concepts and applications to study hepatic effects of food additives and chemicals. Toxic and Applied Pharm 207(2 Suppl):179-188

14. Castoldi M, Vumic Spasic M, Altamura S, Elmen J, Lindow M, Kiss J, Stolte J, Allessandro Sparla R, Klingmuller LA, Fleming RE, Longerich T, Grone HJ, Benes V, Kauppinen S, Hentze MW, Muckenthaler MU (2011) The liver-specific microRNA miR-122 controls systemic iron homeostasis in mice. Clin Invest 121 (4):1386-1396

15. Zhang C, Wang C, Chen X, Yang C, Li K, Wang J, Dai J, Hu Z, Zhou X, Chen L, Zhang Y, Li Y, Qiu H, Xing J, Liang Z, Ren B, Yang C, Zen K, Zhang C-Y (2010) Expression profile of microRNAs in serum: a fingerprint of esophageal squamous cell carcinoma. Clin Chem 56:1871-1879

16. Margis R, Margis R, Rieder CRM (2011) Identification of blood microRNAs associated to Parkinson's disease. J Biotech 152 (3):96-101

17. Dolganiuc A, Petrasek J, Kodys K, Catalano D, Mandrekar P, Velayudham A, Szabo G (2009) MicroRNA expression profile in Lieber-DeCarli diet-induced alcoholic and methionine choline deficient diet-induced nonalcoholic steatohepatitis model in mice. Alc Clin Exp Res 33(10):1704-1710

18. Budhu A, Jia HL, Forgues M, Liu C-G, Goldstein D, Lam A, Zanetti KA, Ye QH, Qin L-X, Croce CM, Tang Z-Y, Wang XW (2008) Identification of metastasis-related microRNAs in hepatocellular carcinoma. Hepatology 47:897-907

19. Li L-M, Hu Z-B, Zhou Z-X, Chen X, Liu F-Y, Zhang J-F, Shen H-B, Zhang C-Y, Zen K (2010) Serum microRNA profiles serve as novel biomarkers of $\mathrm{HBV}$ infection and diagnosis of HBV-positive hepatocarcinoma. Cancer Res 70 (23):9798-9807

20. Yamamoto T, Tomizawa K, Fukikawa M, Sato Y, Yamada H, Horii I (2007) Evaluation of human hepatocyte chimeric mice as a model for toxicological investigation using panomic approacheseffect of acetaminophen on the expression profiles of proteins and endogenous metabolites in liver, plasma and urine. J Toxicol Sciences 32(3):205-215

21. Pagni J (2010) Amroy aims to become nano-leader. European Plastics News. 3-25, March 5, 2010

22. Zanello L, Bin Z, Hui H, Haddon R (2011) Bone cell proliferation on carbon nanotubes. Nanoletters 3(6):562-567, Retrieved 2011-3-25

23. Poland C, Duffen R, Kinloch I, Maynard A, Wallace WAH, Seaton A, Stone V, Brown S, MacNee W, Donaldson K (2008) Carbon nanotubes introduced into the abdominal cavity of mice show asbestos-like pathogenicity in a pilot study. Nature Nanotechnology 3:423-428

24. Teeguarden JG, Robertson B-JW, Waters K, Muarry A, Kisin EL, Varnum SM, Jacobs J, Pounds JG, Zanger R, Shvedova A (2010) Comparative proteomics and pulmonary toxicity of instilled single walled carbon nanotubes, crocidolite asbestos and ultrafine carbon black in mice. Tox Sci 120(1):123-135

25. Mamas M, Warwick BD, Neyses L, Goodacre R (2011) The role of metabolites and metabolomics in clinically applicable biomarkers of disease. Arch Toxicol 85:5-17
26. Ellinger-Zigelbauer $\mathrm{H}$, Adler $\mathrm{M}$, Amberg A, Brandenburg JJ, Callanan S, Connor M, Fountoulakis GH, Gruhler A, Hewitt P, Hodson M, Matheis KA, McCarthy D, Raschke M, Riefke B, Schmitt CS, Siber M, Sposny A, Suter L, Sweatman B, Mally A (2010) The enhanced value of combining conventional and "omics" analyses in early assessment of drug-induced hepatobiliary injury. Toxic and Applied Pharm 252(2):97-111

27. Audouze K, Juncker AS, Roque FJSSA, Krysiask-Baltyn K, Weinhold N, Taboureau O, Jensen TS, Brunak S (2010) Deciphering diseases and biological targets for environmental chemicals using toxicogenomics networks. PLOS Comp Biol 6(5):e1000788

28. Pelley J (2008) Tracking plastics' breakdown products. Environ Sci Tech 42(14):5035-5036

29. Gillum N, Karabekian Z, Swift LM, Brown RP, Kay MW, Sarvayzan N (2009) Clinically relevant concentration of Di (2-ethylhexyl) phthalate uncouple cardiac syncytium. Toxic Applied Pharm 236 (1):25-38

30. Hokenson R, Hanneman W, Hennessey M, Donnelly KC, McDonald T, Chowdhary R, Busbee DL (2006) DEHP (bis-2) ethylhexylphthalate, alters gene expression in human cells: possible correlation with initiation of fetal developmental abnormalities. Hum Exp Toxicol 25(12):687-695

31. Yang L, Milutinovic PS, Brosnan RJ Eger EL (2007) The plasticizer di-(2-ethylhexyl)phthalate modulates gammaaminobutyric acid type A and glycine receptor function. Anesth Analgesia 105(2):393-396

32. Kim SY, Yang JH (2005) Neurotoxic effects of 2,3,7,8 tetrachlorodibenzo-p-dioxin in cerebellar granule cells. Expr Mol Med 37:58-64

33. Craig DGN, Lee A, Hayes PC, Simpson KJ (2010) Review article: the current management of acute liver failure. Aliment Pharmacol Ther 31:345-358

34. Bronstein AC et al (2009) The 2008 annual report of the American Association of Poison Control Centers National Poison Data System (NPDS): 26th annual report. Clin Toxicol 47:911-1084

35. Bond GR, Novak JE (1995) The human and economic cost of paracetamol (acetaminophen) overdose. Pharmacoeconomics 8:177-181

36. Salhanick SD, Shannon MW (2007) Acetaminophen. Haddad and Winchester's clinical managements of poisoning and drug overdose, 4th edn. Elsevier, New York, pp 825-834

37. Rzucidlo SJ, Bounous DI, Jones DP, Brackett BD (2000) Acute acetaminophen toxicity in transgenic mice with elevated hepatic glutathione. Vet Hum Toxicol 42:146-150

38. Laub DN, Elmagbari NO, Elmagbari NM, hausburg MA, Gardiner CS (2000) Effects of acetaminophen on preimplantation embryo glutathione concentration and development in vivo and in vitro. Toxicol Sci 56:150-155

39. Hinson JA, Roberts DW, James LR (2010) Mechanism of acetaminophen-induced liver necrosis. Adverse drug reactions. Handbook of experimental pharmacology. Springer, Heidelberg, pp 369-405

40. Sharma H, Nguyen D, Chen A, Lew V, Khine M (2011) Unconventional low-cost fabrication and patterning techniques for point of care diagnostics. Ann Biomed Eng 39(4):1313-1327 